\title{
Review: evidence on anxiolytic drugs for smoking cessation is inconclusive
}

Hughes JR, Stead LF, Lancaster T. Anxiolytics for smoking cessation. Cochrane Database Syst Rev 2000;(4):CD002849

(latest version 29 Aug 2000).

\section{QUESTION: Do drugs with anxiolytic properties help people to stop smoking?}

\section{Data sources}

Studies were identified by searching Medline, EMBASE/ Excerpta Medica, the Cochrane Tobacco Addiction Group's specialised register, and abstracts from the meetings of the Society for Research on Nicotine and Tobacco.

\section{Study selection}

Studies were selected if they were randomised trials comparing a drug with anxiolytic properties with placebo or an alternative treatment with a follow up $\geqslant 6$ months. Studies on $\beta$ blockers were included, whereas studies on clonidine were not (clonidine is covered in a separate Cochrane review*).

\section{Data extraction}

2 reviewers extracted data on participant characteristics, drug regimens, method of randomisation, completeness of follow up, and results; the outcome was abstinence from smoking assessed at $\geqslant 6$ months of follow up. Reviewers rated the quality of study methods.

\section{Main results}

6 studies met the selection criteria. The studies were done in the US (4 studies), China (1 study), and Scotland (1 study). Sample sizes ranged from 100-324 people (mean 159 people). Treatment with buspirone (2 studies, pooled odds ratio [OR] $0.7,95 \%$ CI 0.3 to 1.5 ), diazepam (1 study, OR 1.0, CI 0.4 to 2.5), or meprobamate (1 study of 3 subgroups with ORs ranging from 0.4 to 0.8 ) did not lead to greater smoking cessation rates than placebo. In the study on diazepam, both groups had behavioural therapy, and abstinence rates were $37 \%$ in each group. One study compared buspirone with transdermal nicotine and reported slightly higher (although not statistically significant) cessation rates for buspirone (OR 1.1, CI 0.6 to 2.1). Another study compared 2 types of $\beta$ blockers with placebo in 101 people; at 12 months, the cessation rates were $17 \%$ for oxprenolol, $24 \%$ for metoprolol, and 3\% for placebo. The difference was statistically significant for metoprolol (OR 5.5, CI 1.4 to 22.4) but not for oxprenolol (OR 4.2, CI 0.9 to 20.2), and this difference developed after the end of drug treatment. No long term studies of ondansetron were found.

\section{Conclusion}

The evidence on the effectiveness of drugs with anxiolytic properties for increasing smoking cessation is inconclusive.
*Gourlay SG, Stead LF, Benowitz NL. Clonidine for smoking cessation. Cochrane Database Syst Rev 2000;(2):CD000058

\section{COMMENTARY}

The Cochrane Library is an electronic goldmine of hundreds of methodologically explicit systematic reviews of medical topics. This review of anxiolytics for smoking cessation by Hughes $e t a l$ is an excellent example of the Cochrane approach to comprehensive systematic reviewing. It focuses on 6 studies that pass methodological muster, but also summarises 9 others that have $<6$ months of follow up or other flaws. The biological rationale for studying anxiolytic medication for smoking cessation is reasonable but not highly compelling. Most anxiolytics have effects on neurotransmitters that are likely involved in nicotine craving. Some evidence shows that anxiety increases in the early phases of smoking cessation and could cause relapse. Views about the role of anxiety in relapse are, however, conflicting. ${ }^{1}$

The authors' conclusion that "the available evidence neither supports nor rules out an effect of anxiolytics on smoking cessation" is justified by the imprecision of both the individual study effect measures (due to small sample sizes) and the limited opportunity to combine study results. Some other tentative conclusions are, however, possible. If we accept that the doses of buspirone, diazepam, and meprobamate used were indeed anxiolytic, the best available evidence argues against the hypothesis that reducing anxiety should increase long term cessation rates. A qualitative overview of all buspirone studies ( 3 short term and 3 long term studies) suggests that a clinical effect on long term cessation larger than that of nicotine replacement is unlikely, but short term effects on cessation and withdrawal are probable. The findings about $\beta$ blockers are interesting but hard to explain biologically, unless these drugs somehow select for smokers who are less likely to relapse. If they were shown to be effective, only buspirone and $\beta$ blockers would have practical potential for use in smoking cessation, so further research on these agents is warranted. Given that several effective medications for smoking cessation are available (bupropion, nortryptiline, and clonidine), current clinical use of buspirone and $\beta$ blockers should probably be limited to smokers who have failed all proven pharmacotherapies and reasonable combinations of the proven treatments (eg, bupropion plus nicotine replacement).

Roger Luckmann, MD, MPH University of Massachusetts Medical School Worcester, Massachusetts, USA

of Vermont, 38 Fletcher 05401-1419, USA.

Fax +1802656 9628 